Association for Information Systems

AIS Electronic Library (AISeL)

Wirtschaftsinformatik 2021 Proceedings

Track 19: IT Strategy, Management \&

Transformation

\title{
Identifying and Overcoming Future Challenges in Leadership: The Role of IS in Facilitating Empowerment
}

Maren Gierlich-Joas

Ludwig-Maximilians-Universität München

Follow this and additional works at: https://aisel.aisnet.org/wi2021

Gierlich-Joas, Maren, "Identifying and Overcoming Future Challenges in Leadership: The Role of IS in Facilitating Empowerment" (2021). Wirtschaftsinformatik 2021 Proceedings. 4.

https://aisel.aisnet.org/wi2021/FITStrategy19/Track19/4

This material is brought to you by the Wirtschaftsinformatik at AIS Electronic Library (AISeL). It has been accepted for inclusion in Wirtschaftsinformatik 2021 Proceedings by an authorized administrator of AIS Electronic Library (AISeL). For more information, please contact elibrary@aisnet.org. 


\title{
Identifying and Overcoming Future Challenges in Leadership: The Role of IS in Facilitating Empowerment
}

\author{
Maren Gierlich-Joas ${ }^{1}$ \\ ${ }^{1}$ Institute for Information Systems and New Media, LMU Munich, Germany \\ gierlich@bwl.lmu.de
}

\begin{abstract}
As digital workplaces change due to innovative technologies, managers have to deal with novel expectations of leadership. In more concrete terms, employees tend to prefer enabling leadership styles over coercive approaches. At the same time, information systems (IS) for leadership get more powerful and are applied in support of leadership. In this study, we investigate both the challenges that arise for leadership because of the changes in framing conditions and how these challenges can be overcome. We carry out an explorative Delphi study to build on the experience of a carefully selected panel of experts. We also gain important insights by conducting qualitative follow-up interviews with specific experts from the panel. The findings emphasize the increasing role of employee empowerment. Organizational change is essential to overcome the challenges, and leadership-related IS can facilitate this transformation to a certain degree. In sum, this study contributes to research on leadership in the digital age.
\end{abstract}

Keywords: Digital transformation, leadership, empowerment, Delphi study

\section{Introduction}

"Upcoming challenges regarding leadership can only be overcome if managers empower their employees." (Consultant1)

Leadership is changing due to the spread of novel technologies and increasing amounts of data. Over the past few decades, leadership has shifted in a more data-centric and employee-focused direction [1]. The use of leadership-related information systems (LRIS) - IS tailored to manage employees on an interpersonal level and to exercise the authority to co-ordinate tasks - makes leadership decisions more objective [2], [3]. These systems have evolved, and their range of functions has drastically expanded [4]. Basic payroll systems from the 1950s evolved into early versions of decision support systems in the 1980s, and sophisticated people analytics solutions have recently been developed [4]. The first systems were mainly designed to facilitate operative tasks in HR, whereas today's solutions support strategic decision making and drive change in leadership [5]. The question is whether this new generation of LRIS helps to master future challenges in leadership.

16th International Conference on Wirtschaftsinformatik,

March 2021, Essen, Germany 
The analysis of past research leads to two major areas of interest: On the one hand, prior research has examined leadership tasks and novel requirements. Scholars have identified employees' shifting values and remote work as triggers for leadership changes [1], [6]. On the other hand, research on LRIS has focused on existing solutions. To a large extent, some technologies already support leaders' tasks effectively. However, biases and information overloads are potential shortcomings hindering the successful transformation in leadership [3], [7]. The gap between the desired and the present system features might even widen due to the changes in organizations' framing conditions and the transformation in leadership styles.

From studying past contributions, we derive a lack of understanding of technologies' role in mastering future challenges for leadership. With this study, we aim to outline future challenges for leadership and approaches to overcome them. Thus, we propose the following research question:

RQ: What are the most important challenges facing the leader of an organization in the coming years regarding novel technologies, and how can they be overcome?

The remainder of the paper is organized as follows: Firstly, we outline the theoretical foundation for the study by introducing the concept of leadership (section 2.1), deriving current trends and technological developments (section 2.2) and presenting control theory as a theoretical lens (section 2.3). Next, we describe the chosen methodological approach - a Delphi study design - by outlining the selection of the expert panel, data collection and data analysis (section 3). The study's findings are presented in section 4, followed by additional insights that we derived from semi-structured interviews with selected experts from the panel to deepen our understanding. We discuss our findings in section 5. Finally, our theoretical and practical contributions are highlighted, limitations are pointed out, and suggestions for further research are listed in section 6. The study offers insights for theory and practice as it contributes to the understanding of future challenges in leadership from a control theory point of view and sheds light on the opportunities to overcome them, partly by using LRIS.

\section{Theoretical Foundation}

\subsection{Concept of Leadership}

Leadership has a long history in the field of management, and definitions vary greatly. Following an extensive literature overview, "[1] eadership has been defined in terms of individual traits, leader behaviour, interaction patterns, role relationships, follower perceptions, influence over followers, influence on task goals, and influence on organizational culture" [8]. One similarity between the definitions relates to one party exerting influence on another party; apart from that, however, the meanings can differ significantly [8]. Most scholars distinguish between "management" and "leadership" by defining management as more task-oriented and leadership as more visionary [9], although the two concepts do overlap in some respects [8]. According to Mintzberg, the "leader" is a specific facet of a manager's roles consist of interpersonal, informational and decisional roles [10]. Leadership itself includes different functions, 
such as composing a team, setting objectives, defining KPIs and measuring progress, building a relationship with employees and managing organizational and cultural ambidexterity [11].

In the context of this work, we define leadership as the management of employee relations and the exercise of authority to co-ordinate tasks within a company to fulfil operative and strategic goals [12]. Leadership has been conceptualized in various leadership theories and leadership concepts. While leadership theories aim to offer explanations for leadership behaviour or to predict future developments, leadership concepts address the implementation of concrete guidelines.

\subsection{Current Trends in Leadership and Leadership-related Information Systems}

In a digital work environment, leadership is subject to change. Driven by the use of novel technologies (technology-push) and the changing needs of employees (technology-pull), leadership approaches increasingly focus on collaboration, empowerment and participation [1].

Regarding the technology-push, the use of IS in HR and leadership has drastically escalated over the past few decades. The aim of IS is to collect, process, store, analyse and disseminate information for a specific purpose [13] - in this case, to support leadership. Hence, we define leadership-related IS (LRIS) as a specific class of IS that are used to support operative and strategic goals inside firms in order to manage employees on an interpersonal level and to exercise their authority to co-ordinate tasks [12]. Thus, we understand LRIS as a combination of strategic management information systems (MIS) and operational human resource information systems (HRIS). MIS are part of LRIS as their purpose is to aggregate and analyse leadership-related data in a data warehouse and to visualize important findings on dashboards so that managers can use data to improve their decision-making abilities [14]. In addition to these strategic planning and control systems, HRIS have emerged as "system[s] used to acquire, store, manipulate, analyse, retrieve, and distribute information regarding an organization's human resources to support HRM and managerial decisions" [15]. For both types of IS, the range of functionalities has been extended significantly since they were first introduced to the market, leading to the chance to facilitate controlling and strategic leadership activities [4]. Integrating insights from operative everyday observations in HR with long-term strategical predictions forms a basis for data-driven leadership approaches.

As for the technology-pull, there is a rising demand for empowerment, which creates a strong interest in LRIS supporting transparency and participation [16]. Once these novel digital solutions are applied in firms, they trigger a transformation on the business side [17]. In times of organizational or technological change, "[1] eadership becomes a very critical element of change management" [18]. Consequently, novel leadership concepts, like shared leadership, which emphasize the role of employees, replace static approaches that put managers in the foreground [6]. Furthermore, leadership has to be tailored to an increasingly digital organization, and digital capabilities have to be built up, which is referred to as "digital leadership" [19]. Similarly, the concept of e- 
leadership describes "leadership in a technology-enabled working environment, leader's competence and the requirements of tasks" [6]. Thus, digital leadership and eleadership refer to leadership in an increasingly digital work setting, in contrast to IT leadership, which describes IT management and is not the focus of this study [20].

\subsection{Leadership from a Control Theory Perspective}

The highlighted trends in leadership change the traditional control styles, which leaders apply and can be examined from a control theory perspective. Control is "any attempt to align individual behaviours with organizational objectives" [21]. Control theory has been transferred from the field of management [22] to IS research and is often used in the context of software development [23]. However, because of its origins, the range of application is much broader and covers both organizational and leadership phenomena [24]. Control theory covers the who, when, why, what and how dimensions of the use of control in an organizational context [24].

The use of control is strongly connected to current leadership approaches. The how dimension, in particular, is of interest for the study, as it describes two distinct control styles: coercive and enabling [25]. Coercive control describes ways of leadership that aim to track employees during task execution [24]. By contrast, an enabling control style aims to "enable employees to better master their tasks" [24] by providing transparency on processes in a way that permits employees to work in a self-organized way. Thus, despite the negative connotation of "control", positive control styles can also be defined as employee-friendly.

Coercive and enabling control styles can be distinguished by four generic principles; repair, internal transparency, global transparency and flexibility [26] (see Table 1). An enabling control style is characterized by a high degree of repair, which helps with employee integration. Enabling control styles have high levels of internal transparency (the understandability of internal processes) and global transparency (employees' involvement in the broader organization). If a control style is flexible and designed to support individual skills, it is labelled as enabling; if flexibility is low, it can be classified as coercive [26].

Table 1. Features of enabling and coercive control styles (following Adler)

\begin{tabular}{lllll}
\hline & Repair & $\begin{array}{l}\text { Internal } \\
\text { transparency }\end{array}$ & $\begin{array}{l}\text { Global } \\
\text { transparency }\end{array}$ & Flexibility \\
\hline Coercive control & low & low & low & low \\
Enabling control & high & high & high & high \\
\hline
\end{tabular}

Both control styles are reflected in different styles of leadership. As prior research shows, employees clearly demand enabling control mechanisms [27]. Hence, modern leadership approaches should satisfy the request for maximal internal and global transparency, the integration of employees and flexible solutions tailored to every individual's needs. In sum, these novel trends in leadership can be interpreted using the various dimensions of control theory. 


\section{Methodological Approach}

We apply a Delphi study to investigate future challenges in leadership from several experts' perspectives. The Delphi study seeks to build consensus between a group of experts on a specific question via a structured process of repetitive questionnaires with controlled feedback [28-29]. In this study, a ranking-type Delphi study is applied to identify relevant factors and reach agreement on their relative importance [28-29]. The structured and anonymous process is suitable to gain insights from the collective experience of experts while avoiding biases that might arise from direct confrontation [30]. Prior contributions demonstrate the fit between similar research questions and the methodology of Delphi studies [31].

The work follows the process established by Schmidt [32], which consists of brainstorming, selection and ranking. In total, four rounds were conducted on a weekly basis between June and July 2020. The study was designed, pre-tested and carried out via an online survey platform that can provide anonymity to the respondents [30]. Throughout the whole process, established quality criteria, were used to ensure the methodological rigour of the study (see Table 2).

Table 2. Attributes used to assess ranking-type Delphi studies (following [30])

\begin{tabular}{llc}
\hline Areas & Attributes & Fulfilled? \\
\hline Research & Follow explicit procedures for expert selection & (search strategy) \\
Design & Use clear selection criteria & $\mathrm{x}$ \\
& Document expert demographics and profiles & $\mathrm{x}$ \\
& Ensure anonymity of participants & $\mathrm{x}$ \\
& Report response rate to initial call & $\mathrm{x}$ \\
& Report panel size & $\mathrm{x}$ \\
& Pretest task instructions and questionnaire & (final design) \\
Brainstorming & Provide clear brainstorming instructions & $\mathrm{x}$ \\
& Ask experts to describe the meaning of items & $\mathrm{x}$ \\
& Have researchers consolidate list of items & $\mathrm{x}$ \\
& Have experts comment and validate list & $\mathrm{x}$ \\
Narrowing & Report final number of items & $\mathrm{x}$ \\
& Provide clear narrowing down instructions & $\mathrm{x}$ \\
& Randomly order list of items & $\mathrm{x}$ \\
& Clearly specify item selection rule & $\mathrm{x}$ \\
& Apply a stopping rule & $\mathrm{x}$ \\
Ranking & Provide clear ranking instructions & $\mathrm{x}$ \\
& Randomly order items (in 1st round) & $\mathrm{x}$ \\
& Ask experts to justify their rankings & $\mathrm{x}$ \\
& Perform appropriate statistical analyses & $\mathrm{x}$ \\
& Apply a stopping rule & $\mathrm{x}$ \\
& Provide controlled feedback to experts & $\mathrm{x}$ \\
\hline
\end{tabular}




\subsection{Panel Selection}

The first step of a Delphi study is the panel selection. The procedure of selecting and inviting the experts was guided by Paré's recommendations for rigorous Delphi studies [30], following principles like clearly defining the selection criteria, documenting the experts' demographics and ensuring the panellists' anonymity.

Firstly, we created a knowledge resource nomination worksheet (KRNW) to derive categories of experts [28]. The KRNW consisted of specialists from industry (suppliers and users of IS for leadership), academia and consultants. Experts from industry are senior-level HR personnel or the leaders of a highly specialized team, e.g. people analytics. They have worked at least two years at a company recently awarded for innovative leadership approaches and use LRIS. Owing to the company sample and the position of the experts within the organization, they are considered suitable for our study. Experts from academia are professors or senior-level researchers at renowned German universities or research institutes and have published research on digital leadership or LRIS in the past three years. Thus, they have a deep knowledge of relevant scientific trends. Consultants were nominated if they work in a consultancy firm specializing in leadership and digital transformation and have at least two years of experience. Because of the clear definition of selection criteria following Pare [30], we assume that we established a qualified panel representing a wide range of perspectives.

Next, the experts were listed and ranked by qualifications. A total of 88 individuals were invited to the study and 23 agreed to participate, which is in line with recommenddations for panel sizes and equals a response rate of $26 \%$ [33]. 17 of these experts (74\%) are male, which we consider a representative distribution, given the background and the positions we sampled for. Furthermore, $61 \%$ of the panellists have an industry background, $17 \%$ do research in the field, and $22 \%$ are consultants (see Table 3 ).

Table 3. Profile of the expert panel

\begin{tabular}{llr}
\hline Characteristics & Panel profile & $(n=23)$ \\
\hline Functional affiliation & Industry & $14(61 \%)$ \\
& Academia & $4(17 \%)$ \\
& Consulting & $5(22 \%)$ \\
Years of experience & Mean & 9.8 years \\
& Min. value & 2 years \\
& Max. value & 20 years \\
Industry & IT & 6 \\
& Consulting & 5 \\
& Academia & 4 \\
& Pharmaceutics & 2 \\
& Mechanical engineering & 1 \\
& Electrical engineering & 1 \\
& Finance & 1 \\
& Telecommunications & 1 \\
& Construction & 1 \\
& Logistics & 1 \\
\hline
\end{tabular}




\subsection{Data Collection and Analysis}

In the following section we outline our data collection, consisting of the different phases of brainstorming, selection and ranking.

Brainstorming. The data collection process (see Table 4) starts with the brainstorming phase, which facilitates the unstructured collection of responses to one (or multiple) open question(s) introduced by the researchers [32]. We posed the initial question: "What are the most important challenges facing the leader of an organization in the coming years regarding the spread of novel technologies and the rising volume of data?"

The experts were asked to name at least five challenges and to describe them briefly in order to increase clarity of their meaning [32]. To achieve a diverse set of initial responses, the number of responses was not limited, in line with the recommendation by Schmidt [32]. The specialists named 114 challenges, which the researchers consolidated by following the guidelines by Paré [30]. The consolidated list of 24 challenges was handed back to the panel for validation to reduce noise and provide further opportunities to receive feedback from the experts [30].

Selection. The selection phase aims to narrow down the consolidated lists obtained via the brainstorming phase to a manageable number of items. The participants were instructed to choose the ten most relevant challenges from the lists, so a concrete number of items was stated [30]. The items were ordered randomly to avoid any biases [32]. Moreover, the validated explanations of the items were displayed during all phases when hovering over the items to create a mutual understanding and avoid noise. The selection was clear-cut, and the items were taken as inputs for the subsequent ranking phase if at least $50 \%$ of the experts had selected them.

Ranking. For the ranking phase, the participants received a fourth questionnaire, which instructed them to rank the shortened list of challenges. For each challenge, the percentage of panel experts who selected the respective value in the previous selection phase was indicated in an anonymous way to equip the panellists with controlled feedback of the panel's evaluation as suggested by Paré [30]. Additionally, we asked for a brief justification of the ranking of the challenges to increase the study's explanatory power [30].

After the first ranking, the mean rank for each item and the Kendall's W coefficient were calculated. Kendall's W is a measure for agreement ranging from 0 (no consensus) to 1 (perfect consensus) [28]. A value of $\mathrm{W}$ greater than 0.7 indicates strong agreement and is often applied as a stopping criterion for the iterative ranking phase [32]. However, before conducting a new round, the trade-off to increase the value of $\mathrm{W}$ and the risk of losing participants has to be considered carefully [32]. Dropout rates between 20 and $30 \%$ are considered normal for Delphi studies [34], but we did not want to endanger the study's findings by adding a fifth round. Thus, the study was closed when a Kendall's W of 0.22 was reached in the fourth round. 
Table 4. Overview of the data collection process

\begin{tabular}{lllll}
\hline & Brainstorming & Selection & Ranking \\
\hline Round & 1 & 2 & 3 & 4 \\
Theme & $\begin{array}{l}\text { Collection of } \\
\text { initial items }\end{array}$ & $\begin{array}{l}\text { Validation of } \\
\text { consolidated } \\
\text { lists }\end{array}$ & $\begin{array}{l}\text { Selection of } \\
\text { top ten items }\end{array}$ & $\begin{array}{l}\text { Ranking of } \\
\text { the final lists }\end{array}$ \\
$\begin{array}{l}\text { Responses } \\
\text { Response Rate }\end{array}$ & 23 & 20 & 20 & 19 \\
\hline
\end{tabular}

\subsection{Additional Data Collection via Follow-Up Interviews}

After completing the Delphi study, we followed the suggestion by Singh et al. to conduct follow-up interviews with selected panellists to add depth to our findings [31]. We approached five experts from the original panel: two male and three female experts; one was working in consultancy, one in academia and three in industry (see Table 5). Building on Myers and Newman, we prepared guidelines for the semi-structured interviews [35]. In the interviews, we asked the experts to elaborate on 1) the main challenges from the Delphi study, 2) ways to overcome them and, 3) more specifically, the role of LRIS in overcoming them. The interviews were conducted via videoconferencing tools between October and November 2020 and lasted between 25 and 40 minutes. The participants' anonymity was guaranteed during the whole process, and feedback from the earlier Delphi study was provided. The interviews were recorded, transcribed and then analysed with the software Atlas.ti following iterative rounds of coding as suggested by Miles et al. [36].

Table 5. Overview of panellists for follow-up interviews

\begin{tabular}{llll}
\hline Pseudonym & Industry & Experience(years) & Gender \\
\hline Provider1 & IT & 10 & Female \\
Provider2 & IT & 4 & Male \\
User10 & Mechanical engineering & 5 & Female \\
Consultant4 & Consultancy & 17 & Female \\
Academic1 & Academia & 6 & Male \\
\hline
\end{tabular}

\section{$4 \quad$ Findings}

\subsection{Findings Regarding Leadership Challenges}

In the brainstorming session, numerous leadership challenges were collected in connection with digital transformation. Table 6 illustrates the findings of the selection and ranking phase for the challenges ordered by their rank after the fourth round, including the experts' definitions. 
Table 6. Findings of the selection and ranking phase for leadership challenges

\begin{tabular}{|c|c|c|c|}
\hline Challenge & $\begin{array}{l}\text { Selection } \\
\text { share of } \\
\text { experts who } \\
\text { selected the } \\
\text { challenge }\end{array}$ & $\begin{array}{l}\text { Ranking } \\
\text { mean rank }\end{array}$ & Rank \\
\hline $\begin{array}{l}\text { Empowerment of employees: hand responsibility to } \\
\text { employees and refrain from strict hierarchies. }\end{array}$ & $65 \%$ & 3.63 & 1 \\
\hline $\begin{array}{l}\text { Digital transformation and organizational change: } \\
\text { lead employees in times of digital transformation. }\end{array}$ & $75 \%$ & 3.68 & 2 \\
\hline $\begin{array}{l}\text { Innovation culture: foster a culture of learning that } \\
\text { benefits from innovations in leadership in reverse. }\end{array}$ & $70 \%$ & 4.21 & 3 \\
\hline $\begin{array}{l}\text { Purposeful leadership: provide meaningful goals to } \\
\text { employees. }\end{array}$ & $65 \%$ & 4.84 & 4 \\
\hline $\begin{array}{l}\text { Individual leadership: address individual needs } \\
\text { instead of applying a "one-size-fits-all" approach. }\end{array}$ & $60 \%$ & 5.21 & 5 \\
\hline $\begin{array}{l}\text { Digital competences: build up knowledge on the use of } \\
\text { novel technologies. }\end{array}$ & $60 \%$ & 5.42 & 6 \\
\hline $\begin{array}{l}\text { Remote leadership: lead and motivate teams from a } \\
\text { distance. }\end{array}$ & $65 \%$ & 6.58 & 7 \\
\hline $\begin{array}{l}\text { Agile methods: lead teams with less clearly structured } \\
\text { hierarchies and shift responsibilities. }\end{array}$ & $50 \%$ & 7.05 & 8 \\
\hline $\begin{array}{l}\text { Volatile environment: adapt leadership to a } \\
\text { dynamically changing environment. }\end{array}$ & $50 \%$ & 7.16 & 9 \\
\hline $\begin{array}{l}\text { Ambidexterity: manage tensions between the core } \\
\text { business and novel innovations in leadership. }\end{array}$ & $55 \%$ & 7.21 & 10 \\
\hline
\end{tabular}

When contrasting the different subgroups of the panel by academia vs. industry vs. consulting or by manager perspective vs. employee perspective, the mean ranks for the items do not differ much. However, managers ranked "digital transformation and organizational change" first and "empowerment of employees" third, whereas employees prioritized "empowerment". The Kendall's W values for the different subgroups do not differ greatly and range between 0.21 and 0.36 , so the level of agreement is similar for the different groups. Below, the top three challenges are outlined.

Challenge \#1 - Empowerment of employees. The approach of handing responsibility to employees and refraining from strict hierarchies was ranked first. One panellist stated that "leadership should be a social participation process" (Academic1). Empowerment can lead to "an abolition of leaders in a traditional way [...] but it challenges employees as they need to take responsibility" (Academic2). Overall, empowerment is considered a key factor because "upcoming challenges [...] can only be overcome if managers empower their employees" (Consultant1).

Challenge \#2 - Digital transformation and organizational change. The panellists defined the challenge as "leading employees in times of digital transformation"; for this reason, they strongly refer to the concept of digital leadership. Since "business models 
change drastically, internal organizational change is a logical consequence" (User4). Therefore, "capabilities that did not exist before rise in importance" (User 2).

Challenge \#3 - Innovation culture. The third-placed challenge is "innovation culture", meaning the ability to "foster a culture of learning that benefits from innovations in leadership in reverse". The definition highlights the understanding that innovations are enabled by a certain culture and leadership style. "Innovation culture is strongly related to individual leadership styles [...] that drive transformational change" (User 12). Since shaping an organization's culture is one of the tasks of its leaders [11], creating a culture of innovation is viewed as a crucial challenge to remain competitive.

\subsection{Enhancing Findings with Results from Follow-Up Interviews}

Guided by the insights from the follow up interviews, we derived more in-depth findings on empowerment as challenge and ways to overcome this obstacle.

Empowerment as a challenge. Discussing the challenge of empowerment in depth led to insights regarding its perceived importance. The experts agreed: "codetermination is an important topic in many firms" (Provider2). "It sets the framing conditions for employees to master digital transformation as it provides opportunities to shape their environment" (Consultant4). Thus, while empowerment is seen as a game-changer for leadership in the digital age, it comes with certain challenges.

For example, defining empowerment in practice seems to raise questions, as "a major challenge is to develop a model of what empowerment actually is" (Provider2). The concept "seems to be too fuzzy and people understand different things" (Academic1). The scope of empowerment needs to be defied in terms of "who is empowered, when, for which reason and up to which degree?" (Academic1). Thus, starting initiatives for empowerment is difficult if clear objectives are lacking.

Once the term "empowerment" is clarified, its implementation has to be conducted thoroughly. Enforcing empowerment might lead to mistrust: "I was used to doing everything my way, and suddenly everything becomes transparent - I don't like that" (Consultant4). In this scenario, empowerment can be interpreted as control instead of a chance for self-organization, which "leads to great negative outcomes" (Academic1) and which has to be avoided to keep employee satisfaction high. And even if the goals for empowerment are clarified, "the organizational structure and culture can be burdens" (Academic1).

Overcoming the challenge of empowerment. Our selected experts outlined a few solutions to overcome the challenge of empowerment from a non-technical perspective (see Table 7). Firstly, establishing a culture of trust and a mindset of supportive leadership is considered crucial; otherwise, measures to increase empowerment might be interpreted as control. Employees need to have incentives to trust empowerment initiatives and related LRIS. The novel organizational mindset goes hand in hand with a changed understanding of leadership. As decision making can be supported by LRIS, "leaders can invest more time in caring for their employees, developing them" (Consultant4).

Secondly, organizations have to establish transparency to reach empowerment. “Transparency is key to empowering employees, as those who don't have access to data 
and don't see the big picture can't make decisions wisely" (Provider1). "By showing positive and negative use-case scenarios [of LRIS] in a transparent way, acceptance can be increased" (Provider2). Furthermore, companies have to "prove that tracking mechanisms are not applied" (Provider2). To increase trust, transparency needs to be implemented at all organizational levels, and experts are "mystified as to why employees should become fully transparent when companies aren't disclosing their data" (Provider1). They demand a reciprocal model of transparency that grants both managers and employees access to the data.

Lastly, digital capabilities need to be built up to facilitate the use of LRIS. "Employees in IT-related environments are happy with the systems, but for employees in production, [...] the manager is in charge of using the tools" (Provider1). Employees need to be permitted to take over responsibility and use these systems independently.

The role of LRIS in overcoming the challenge of empowerment. Additionally, the experts outlined ways in which technology can facilitate empowerment, "as structures and data become visible" (Provider1). Some of the system functionalities were named that help to increase empowerment and transparency (see Table 7).

Firstly, LRIS help to define empowerment and measure the success of empowerment initiatives. As employee surveys can be conducted digitally every week, "they give leaders an important overview regarding mood, motivation and feedback" (Consultant4). Via structured feedback routines, KPIs for empowerment can be displayed on charts to illustrate their long-term development.

Secondly, LRIS assist in generating transparency as a basis for empowerment. Applying the "principles of user design controlling [...] to visualize insights in comprehensive ways, e.g. by using traffic light notifications" (Provider2), facilitates overall transparency. Customized dashboards for each employee or manager should display the individuals' progress, as well as the teams' working status (User10). Performance measures can be documented and taken as a reference for staff appraisals.

Moreover, using training sessions of LRIS enhances employees' digital capabilities: "on-site trainings that are tailored to the individual stakeholder groups are essential", so employees can convert their opportunities for engagement into actual self-organized work routines (User10). Thus, technology can support the process of developing digital capabilities.

Last but not least, LRIS help to enforce data protection regulations by depicting different user roles with different degrees of data access. However, the experts disagreed on the conceptualization of the different user roles. While one stated that "management should be able to see and compare more data [than the employees]" (Provider10), another explained that "every team member and team leader should have access to all data, [following the principle of] reciprocal transparency" (Consultant4).

In sum, the panel mostly viewed the use of LRIS to overcome the challenge of empowerment in an optimistic light. One expert even stated: "Every task that does not require human intelligence can be undertaken or supported by technical systems" (Academic2). However, the panellists mostly agreed that the role of technology in overcoming the challenge of empowerment is limited. "Technology can also get in the way [of empowerment]" as the tools might replace talks between leader and employee but cannot fully cover the interpersonal level, which leads to misunderstandings 
(User10). Thus, LRIS drive empowerment initiatives but only to a certain degree. "Digital innovations in the HR context can help in overcoming certain challenges but often we expect too much [...]. The way we empower employees is strongly driven by daily interactions which cannot be replaced by technologies" (Acadmic1). Along with the technological solution, the organizational side has to adapt as well which is highly context-specific: "Saying 'we have a great tool' is not enough." (Consultant4).

Table 7. Approaches for overcoming the challenge of empowerment

\begin{tabular}{ll}
\hline Overcoming the challenge & The role of LRIS \\
\hline Define empowerment and set KPIs to & - Introduce regular surveys \& metrics to \\
track initiatives & measure empowerment \\
Create transparency for work processes & - Integrate customized dashboards to \\
across all organizational levels & monitor work processes \\
& - Use LRIS for performance assessment \\
Build up digital capabilities to use LRIS & - Make use of training sessions for LRIS \\
& - Stick to intuitive user interfaces \\
Establish a culture of trust by redefining & - Limited support by LRIS (as it mainly \\
leadership & needs organizational change) \\
& - Define distinct user roles to ensure data \\
& protection and enhance trust \\
\hline
\end{tabular}

\section{Discussion}

The study's findings can be summarized in two major points:

Firstly, leadership's shift towards enabling styles entails novel challenges. Control theory is very suitable to investigate these challenges and we heeded previous calls to apply control theory at the interface of leadership to benefit from its wide span of application [23]. In light of control theory, the top-ranked challenges reflect an enabling leadership approach. The four principles of enabling control styles - namely, repair, internal transparency, global transparency and flexibility (see Table 1) — are present in the challenges cited by the panellists. By contrast, challenges that reveal a clearly coercive approach to leadership, like "transparency on performance measurement" or "monitoring of employees", were named in the brainstorming phase but not chosen in the selection phase. Thus, the experts agreed on the overall trend towards employeecentric, enabling leadership approaches. As recent studies in the field of control theory highlight, the novel degree of transparency in organizations can be used to either enable or track employees [25], [37]. Thus, the thorough implementation of transparency is of high importance as it lays the foundation to prevent mistrust and enables ways of successful empowerment [16]. By examining the challenge of empowerment in an explorative way, we add to the literature on leadership in the digital age and control theory [1], [24]. We find that enabling leadership styles can only be implemented successfully if challenges to organizational culture and the use of LRIS are overcome. 
Secondly, novel LRIS assist in overcoming empowerment as a future challenge in leadership. As the systems provide transparency and offer ways to measure empowerment and employee performance, they strongly drive digital leadership. Scholars have investigated the evolution of IS in the field of HR, which depicts the change from supporting basic HR function to facilitating strategic decision making [4]. Many studies illustrate how HRIS can support recruiting processes, performance evaluation or workforce planning [38]. However, we suggest that novel systems reach even further and can support leadership. Unlike HRIS, LRIS have a strategic orientation and, thus, make it easier to overcome leadership challenges like empowerment.

However, standalone tools will not be sufficient to overcome the mentioned challenges and drive digital leadership approaches. Technological and organizational changes need to go hand in hand. This phenomenon has been investigated with the concept of "technochange" - the strategic use of IT to derive organizational benefits by integrating IT introduction and complementary organizational changes to manage digital culture change via the introduction of IT [39]. This concept supports our findings, as LRIS are implemented for the strategic purpose of changing leadership. However, complementary organizational change is essential to drive digital leadership.

\section{Conclusion}

\subsection{Theoretical and Practical Contribution}

In the study, we investigated future challenges in leadership through the lens of control theory. The Delphi study and the follow-up interviews with carefully selected experts shed light on the obstacles that can be expected, including empowerment, digital transformation and innovation culture. In addition, it is possible to map the challenges to enabling leadership styles. Implementing LRIS with a complementary change in organizational culture can help to overcome the particular challenges. In summary, the study serves as a stepping stone for research on digital innovation in leadership.

Firstly, the study contributes to an understanding of the emerging challenges for leadership in a digital work context. Coming from a management-oriented perspective, we outline challenges for leadership. Next, we provide solutions from a more technology-focused perspective and clarify the role of IS in overcoming the mentioned burdens. In this way, the study aims to bridge the gap between research on designoriented IS and research on management-oriented HR [1].

Secondly, with our study, we emphasize the growing importance of LRIS in driving digital transformation in organizations. In contrast to previous studies [4], we highlight the systems' option to facilitate strategic leadership topics and not only operational HR processes. LRIS can democratize power by providing transparency for employees and are, therefore, key to creating empowerment.

Thirdly, the traditional way of conducting Delphi studies was extended as suggested by several scholars, e.g. Schmidt et al. [33] and Singh et al. [31]. Instead of limiting ourselves to collecting and prioritizing challenges (understanding the problem), we examine solutions through semi-structured interviews (solving the problem). 
From a practical point of view, the study provides novel insights on upcoming leadership trends, related challenges and requirements for LRIS. We offer insights to managers regarding how leadership might change in the digital age and how using LRIS can facilitate this transformation. Moreover, following the outlined challenges, LRIS providers can develop their solutions according to the future needs of the market.

\subsection{Limitations and Outlook}

Although the study was very thorough, our research did have certain limitations. Some of these limitations concern the application of the Delphi study (1), while others involve the general research setting (2).

Firstly, concerning the panel, it is important to note that Delphi studies do not require a representative sample following statistical assumptions [30]. Nonetheless, it might be difficult to draw general assumptions from a relatively small sample that has a high degree of innovativeness. We tried to address this potential shortfall by investigating a diverse sample; however, it should be noted that leadership is highly related to external factors (e.g. culture) that could not be controlled. Furthermore, the level of consensus is relatively low (Kendall's W of 0.22), and a higher degree would have been favourable. Still, as Paré states, as long as appropriate stopping rules are applied, the study's validity does not necessarily suffer from a small degree of agreement [30].

Secondly, concerning the research setting, the Delphi study is a helpful tool to answer "what could/should be" questions, but the explanatory detail that can be expected in qualitative studies is limited. Multiple fields for open comments in the survey addressed this limitation, but only to a limited degree. Therefore, semistructured qualitative interviews with selected experts from the panel added depth to the findings and helped to derive solutions for the listed challenges. However, specific design requirements for future LRIS remain a topic for further investigation. Moreover, the stated challenges and options to overcome them are highly subjective. Owing to the explorative approach, the items do not necessarily follow the mutually exclusive and collectively exhaustive principle. Thus, some challenges might overlap while there were other important factors that the panellists did not mention.

Despite these issues, we consider this study an important starting point for promising future research. Regarding the application of the method (1), adding more rounds of ranking might help to increase the value of the Kendall's W. Future researchers are encouraged to investigate larger samples and to contrast panels with different cultural backgrounds. In addition, we recommend enhancing the research setting (2). To structure the statements from the panellists and avoid missing out on relevant aspects, we suggest contrasting the empirical findings with existing literature. Adding insights from previous scholars after the initial brainstorming phase can be a solid approach to increase the robustness of the findings. Furthermore, we consider Delphi studies a promising foundation for design science research projects as they are an instrument to define the objectives of a solution and to derive design requirements for technical and organizational artefacts [40]. Thus, applying the learnings from this study to a design science research project can pave the way for design-oriented research on digital innovation in leadership. 


\section{References}

1. Cortellazzo, L., Bruni, E., Zampieri, R.: The Role of Leadership in a Digitized World: A Review. Frontiers in Psychology 10, 1-21 (2019)

2. Gierlich, M., Hess, T.: Towards an Understanding on Datas' Influence on Leadership. In: Internationale Tagung der Wirtschaftsinformatik (2020)

3. Tursunbayeva, A., Di Lauro, S., Pagliari, C.: People analytics-A scoping review of conceptual boundaries and value propositions. International Journal of Information Management 43, 224-247 (2018)

4. Johnson, R.D., Lukaszewski, K.M., Stone, D.L.: The Evolution of the Field of Human Resource Information Systems: Co-Evolution of Technology and HR Processes. Communications of the ACM 38, 533-553 (2016)

5. Wirtky, T., Laumer, S., Eckhardt, A., Weitzel, T.: On the Untapped Value of e-HRM: A Literature Review. Communications of the ACM 38, 20-83 (2016)

6. Dinh, J.E., Lord, R.G., Gardner, W.L., Meuser, J.D., Liden, R.C., Hu, J.: Leadership theory and research in the new millennium: Current theoretical trends and changing perspectives. The Leadership Quarterly 25, 36-62 (2014)

7. Gal, U., Blegind Jensen, T., Stein, M.-K.: Breaking the vicious cycle of algorithmic management: A virtue ethics approach to people analytics. Information and Organization 30, 1-15 (2020)

8. Yukl, G.A.: Managerial leadership: A review of theory and research. Journal of Management 15, 251-289 (1989)

9. Zaleznik, A.: Managers and Leaders: Are They Different? Havard Business Review 82, 74 81 (1977)

10.Mintzberg, H.: The manager's jobs: Folklore and fact. In: Vecchio, R.P. (ed.) Leadership, pp. 49-61. University of Notre Dame Press, Notre Dame, Indiana (2007)

11.Morgeson, F.P., DeRue, D.S., Karam, E.P.: Leadership in Teams: A Functional Approach to Understanding Leadership Structures and Processes. Journal of Management 36, 5-39 (2009)

12.Beare, H., Caldwell, B., Millikan, R.: Dimensions of leadership. In: Crawford, M., Kydd, L., Riches, C.R. (eds.) Leadership and teams in educational management, pp. 24-39. Open Univ. Press, Buckingham (1997)

13.Rainer, R.K.T., E.; Potter, R. E.: Introduction to Information Systems. John Wiley \& Sons, Hoboken, NJ (2007)

14.Gorry, A.M., Michael S.S.: A Framework for Management Information Systems. Management Sloan Review 13 (1971)

15.Kavanagh, M.J., Johnson, R.D. (eds.): Human resource information systems: Basics, applications, and future directions. Sage, Los Angeles (2018)

16.Gierlich-Joas, M., Hess, T., Neuburger, R.: More Self-Organization, More Control - Or Even Both? Inverse Transparency As A New Digital Leadership Concept. Business Research forthcoming (2020)

17.Wiesböck, F., Hess, T.: Digital innovations - Embedding in organizations. Electronic Markets 30, 75-86 (2020)

18.Nadler, D.A., Tushman, M.L.: Beyond the Charismatic Leader: Leadership and Organizational Change. California Management Review 32, 77-97 (1990) 
19. Legner, C., Eymann, T., Hess, T., Matt, C., Böhmann, T., Drews, P., Mädche, A., Urbach, N., Ahlemann, F.: Digitalization: Opportunity and Challenge for the Business and Information Systems Engineering Community. Business \& Information Systems Engineering 59, 301-308 (2017)

20.Hoving, R.: Information Technology Leadership Challenges - Past, Present, and Future. Information Systems Management 24, 147-153 (2007)

21.Kirsch, L.J.: The Management of Complex Tasks in Organizations: Controlling the Systems Development Process. Organizational Science 7, 1-21 (1996)

22.Ouchi, W.G.: The Relationship Between Organizational Structure and Organizational Control. Administrative Science Quarterly 22, 95-113 (1977)

23.Cram, W.A.: Information Systems Control: A Review and Framework for Emerging Information Systems Processes. Journal of the Association for Inofrmation Systems 17, 216-266 (2016)

24.Cram, W.A., Wiener, M.: Technology-mediated Control: Case Examples and Research Directions for the Future of Organizational Control. Communications of the Association for Information Systems 46, 70-91 (2020)

25.Heuman, J., Wiener, M., Remus, U., Mähring, M.: To coerce or to enable? Exercising formal control in a large information systems project. Journal of Information Technology 30, 337351 (2015)

26.Adler, P.S., Borys, B.: Two Types of Bureaucracy: Enabling and coercive. Administrative Science Quarterly 41, 61-89 (1996)

27.Carasco-Saul, M., Kim, W., Kim, T.: Leadership and Employee Engagement: Proposing Research Agendas Through a Review of Literature. Human Resource Development Review 14, 38-63 (2015)

28.Okoli, C., Pawowski, S.D.: The Delphi method as a research tool: an example, design considerations and applications. Information \& Management 42, 15-29 (2004)

29.Skinner, R., Nelson, R.R., Chin, W.W., Land, L.: The Delphi Method Research Strategy in Studies of Information Systems. Communications of the ACM 37, 31-63 (2015)

30.Paré, G., Cameron, A.-F., Poba-Nzaou, P., Templier, M.: A systematic assessment of rigor in information systems ranking-type Delphi studies. Information \& Management 50, 207 217 (2013)

31.Singh, R., Keil, M., Kasi, V.: Identifying and overcoming the challenges of implementing a project management office. European Journal of Information Systems 18, 409-427 (2009)

32.Schmidt, R.C.: Managing Delphi Surveys Using Nonparametric Statistical Techniques. Decision Sciences 28, 763-774 (1997)

33.Schmidt, R.C., Lyytinen, K., Keil, M., Cule, P.: Identifying Software Project Risks: An International Delphi Study. Journal of Management Information Systems 17, 5-36 (2001)

34.Bardecki, M.J.: Participants' Response to the Delphi Method: An Attitudinal Perspective. Technological Forecast and Social Change 25, 281-292 (1984)

35.Myers, M.D., Newman, M.: The qualitative interview in IS research: Examining the craft. Information and Organization 17, 2-26 (2007)

36.Miles, B.M., Huberman, A.M., Saldana, J.: Qualitative Data Analysis: A Methods Sourcebook. SAGE Publications Ldt (2013)

37.Liu, G.H., Chua, C.E.: The Reinforcing Effects of Formal Control Enactment in Complex IT Projects. Journal of the Association for Information Systems 21, 312-340 (2020) 
38.Gal, U., Blegind Jensen,s T., Stein, M.-K.: People Analytics in the Age of Big Data: An Agenda for IS Research. In: Proceedings of the Annual International Conference on Information Systems, pp. 1-11. Association for Information Systems, (2017)

39.Markus, M.L.: Technochange Management: Using IT to Drive Organizational Change. Journal of Information Technology 19, 4-20 (2004)

40.Kloör, B., Monhof, M., Beverungen, D., Braäer, S.: Design and evaluation of a model-driven decision support system for repurposing electric vehicle batteries. European Journal of Information Systems 27, 171-188 (2018) 